\title{
A Case Series Outlining the Relationship between Dolichoectasia and Ectrodactyly-Ectodermal Dysplasia-Clefting Syndrome
}

\author{
Cameron John Sabet*
}

University of Pennsylvania, USA

*Corresponding author: Cameron John Sabet, University of Pennsylvania, USA

Ectrodactyly-Ectodermal

Dysplasia-Clefting Syndrome $(E E C)$ is a rare heritable condition marked by ectodermal dysplasia with nails, teeth, sweat glands, and hair, eye and lacrimal duct malformations, midfacial hypoplasia, ectrodactyly (loss of fingers or toes), syndactyly, clinodactyly, auricular anomalies, short height, orofacial cleft palates, genitourinary anomalies, malformations within the central nervous system (CNS) leading to mental impairment or hearing loss, nevocellular nevi, flat noses, and hypopigmentation $[1,2]$. The connection between dolichoectasia and EEC has not yet been outlined in a formal literature review or even case series, so this article aims to explore a series of case reports to better highlight the various etiologies, symptoms, and presentations of EEC as they relate to dolichoectasia.

The dilation and elongation of arteries in the cranial cavity is called cerebral arterial dolichoectasia. To date, the correlation between dolichoectasia and EEC has not been studied thoroughly, though one case report specifically mentioned the possibility of those two conditions being frequently paired without delving into the literature to verify this claim. EEC, specifically lobster claw, occurs both in a familial inheritance pattern as well as sporadically.

One of the earliest articles suggesting a correlation between dolichoectasia and EEC was a paper describing a 44-year-old black female who was born with bilateral lobster claw deformities on her feet, but no other structural abnormalities. Her two siblings had experienced seizures, but no structural abnormalities. This 44-year-old had very little body hair and presented with decayed teeth. For four years before her diagnosis, she had been treated for chronic renal failure and hypertension, as well as having had congestive heart failure many times, each time requiring her admittance to the hospital. At birth, the patient presented with lobster claw deformities of both feet but no other congenital abnormalities. She later developed lancinating pain in her right mandible that was sensitive to movement-chewing or talking mainly.

Eventually, this pain grew to the point that she could not eat or speak at all. Vertebrobasilar angiograms revealed dilation, elongation, and displacement of the basilar artery [3].

All of the case reports describing EEC patients, even the ones from before angiograms were incorporated into the diagnostic checklist for clinicians, agree on some form of mental divergence of the patients afflicted with EEC. For example, one report mentioned "moderate psychomotor" dysfunction, another patient was reported to be placed in an institution for mentally divergent people, another presented with peripheral facial nerve palsy, while yet another case presented with an $81 \mathrm{IQ}$ score $[4,5]$.

The presentation of EEC in relation to dolichoectasia is extremely rare not only due to the fact that each separate condition is already rare, but that EEC is usually attributed to some familial or sporadic inheritance while dolichoectasia usually develops in the presence of longstanding hypertension [6]. We hope that this article better outlines the current literature surrounding the relationship between EEC and dolichoectasia, as well as highlights the lack of understanding arising from the

Citation: Sabet CJ (2021) A Case Series Outlining the Relationship between Dolichoectasia and Ectrodactyly-Ectodermal Dysplasia-Clefting Syndrome. Int Arch Vasc Med 4:010. doi.org/10.23937/26903164/1710010

Accepted: July 29, 2021: Published: July 31, 2021

Copyright: (C) 2021 Sabet CJ. This is an open-access article distributed under the terms of the Creative Commons Attribution License, which permits unrestricted use, distribution, and reproduction in any medium, provided the original author and source are credited. 
absence of angiograms performed before 1970 on EEC patients due to the fact that the technology had not been developed yet.

\section{References}

1. National Foundation for Ectodermal Dysplasias (2021) Ectrodactyly-ectodermal dysplasia-clefting syndrome.

2. Cyriac MJ, Lashpa E (2006) Lobster-claw hand: A manifestation of EEC syndrome. Indian $\mathrm{J}$ Dermatol Venereol Leprol 72: 54-56.

3. Miner ME, Rea GL, Handel S, Bertz J (1980) Trigeminal neuralgia due to dolichoectasia: Angiographic and CT findings in a patient with the EEC syndrome. Neuroradiology 20: $163-166$.

4. Cockayne EA (1936) Cleft palate-lip, hare lip, dacryocystitis, and cleft hand and foot. Biometrika 28: 60-63.

5. Berndorfer A (1969) Gesichtsspalten Gemeinsam Mit hand und Fuß Spalten. Z Orthop 107: 344.

6. Smoker WR, Corbett JJ, Gentry LR, Keyes WD, Price MJ, et al. (1986) High-resolution computed tomography of the basilar artery: 2. Vertebrobasilar dolichoectasia: Clinicalpathologic correlation and review. AJNR Am J Neuroradiol 7: 61-72. 